\title{
Track Following Control Using Nonlinear Model Predictive Control in Hard Disk Drives
}

\author{
M. Taktak-Meziou, A. Chemori, J. Ghommam, and N. Derbel
}

\begin{abstract}
In this paper, a Nonlinear Model Predictive Control (NMPC) is proposed to control a single stage Voice-CoilMotor (VCM) of a Hard-Disk-Drive (HDD). Due to its fast settling time and its robustness, this controller is suggested to be applied for the first time to control a $\mathrm{R} / \mathrm{W}$ head of an HDD. To highlight the good performance and characteristics of the NMPC, a comparative study with a standard PID control is presented. The two control methodologies were evaluated in nominal conditions as well as in other situations such as disturbances and uncertainties on the plant model parameters. In all cases, NMPC presents much better simulation results in term of speed and robustness in the presence of unexpected perturbations and parameters' change.
\end{abstract}

\section{INTRODUCTION}

Research on Hard-Disk-Drives (HDDs) has undergone significant development in recent years. The HDD assembly consists of several rotating disks called platters driven by a spindle motor witch ensures their quick rotation. The data are read from, or written onto, the platters by means of an electromagnetic Read/Write (R/W) head. A Voice-CoilMotor (VCM) ensures the displacement of the head to follow precisely a desired destination track. A view of a typical HDD is illustrated in Fig. 1.

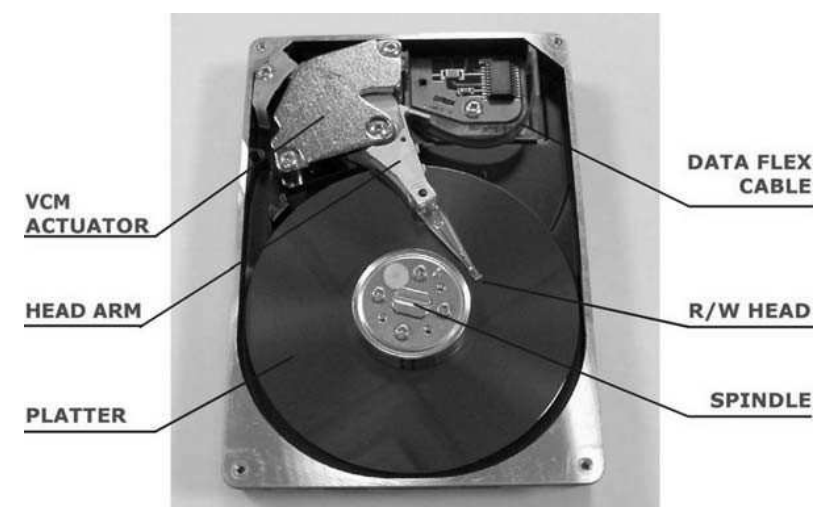

Fig. 1: View of typical components of HDD[15]

The main objective of the HDD servo control system is to maintain the $\mathrm{R} / \mathrm{W}$ head precisely as close as possible to the desired track of the disk (track-following function) while information is being written or read, and to move it

M. Taktak-Meziou, J. Ghommam, and N. derbel are with the ICOS, Departement of Electrical Engineering, National School of Engineers of Sfax (ENIS), BP, W 3038 Sfax Tunisia. (e-mail: manel.taktak@yahoo.com, jawhar.ghommam@gmail.com,n.derbel@enis.rnu.tn)

A. Chemori is with the LIRMM, University Montpellier 2 / CNRS, UMR5506, CC477, 161 rue Ada, 34095 Montpellier Cedex 5, France (email: Ahmed.Chemori@lirmm.fr) from the present track to an another specified track (trackseeking function) to browse all the information subscribed on the disk. This latter function should be achieved in a minimum time using a bounded control algorithm. In this paper, we are interested in the servo controller of the trackfollowing function. It is quite clear that accuracy, rapidity, and robustness are the most demanding specifications to satisfy in the HDD servo control system. Nevertheless, the head of an HDD is a responsive component which cannot be easily kept stable while reading or writing data. External vibrations and chocks are the main factors influencing the performance of the servo-system through increasing the position error of the $\mathrm{R} / \mathrm{W}$ head. In addition, due to the rigidity of the HDD system, the movement of the head from one track to its neighborhood tracks (track-following mode) yields the generation of large residual errors. More details about this fact are sited in section II.

Due to the system itself and its working conditions, different challenges can be found in the literature arising the control of HDDs. The main purpose is to build a robust control scheme meeting the different specifications stated before and leading to a smaller error in the positioning of the head. Previous literature works can be mainly classified into two major categories: The first category contains repetitive approaches which aim to achieve the tracking of a periodic path, the rejection of periodic disturbances, or both [1][2][3]. The second category gathers non-repetitive methods such as robust approaches including $H \infty$ control [4][5], sliding mode control [7] and adaptive control [11], modern approaches like LQG control [6] and classical approaches such as notch filters, PID controller and lead-lag compensators [8][9][10]. Recently, some proposed solutions used prediction based scheme for fast seeking control [12][13].

This paper deals with the application of NMPC approach to control the R/W head of the HDD servo system. Recently, this control technique has gained an increasing interest from different research communities and was applied for the control of a large variety of systems. These applications include robotics [16], aerospace [17] and chemical fields [18]. An overview of NMPC technique is given in [14]. The reasons behind the choice of this control technique are mainly its robustness and ability to meet different constraints and specifications on the systems' variables as well as on the control input. In this paper, NMPC controller is implemented on the HDD servo system in various situations ranging from nominal case to different conditions including disturbances and uncertainties. To the best knowledge of the authors, this control scheme was never been conducted before on a HDD 
servo systems.

The outline of this paper is as follow: section II deals with the description of the system and its dynamic modeling. Section III presents the proposed controller design. The simulation results are presented and discussed in section IV. Finally, concluding remarks are addressed in section $\mathrm{V}$.

\section{SYSTEM DESCRIPTION AND DYNAMICS}

In this section, the modeling of a VCM actuator is presented. The fast displacement of the head from one track to another and its ability to remain in neighborhood of the target destination track give rise to frictional forces and different nonlinearities. As illustrated in Fig.1, the R/W head is connected to a data flex cable. This latter generates undeniable nonlinearities while its expansion and contraction action during the movement of the head. The pivot and bearing frictions are the primarily sources of nonlinearities at low frequencies. All these factors generate large positioning errors and may degrade the performance of the overall system. Therefore, it should be necessary to take into consideration all these nonlinearities when modeling the VCM-actuated HDD servo system.

The mathematical model of the VCM actuator is given by

$$
M \ddot{y}+F(y, \dot{y})=u
$$

where $M$ is the system inertia. $y, \dot{y}$ and $\ddot{y}$ denote respectively the position, velocity and acceleration of the VCM-actuator head tip. $u$ is the control input and $F(y, \dot{y})$ is a nonlinear function representing bias forces due to pivot friction and the flex-cable. Based on the detailed modeling and frequency response identification at low frequencies given in [15], the nonlinear model of the VCM actuator can be expressed as follows:

$$
\begin{gathered}
\ddot{y}=2.35 \times 10^{8} u-6.7844 \times 10^{6} \arctan (0.5886 y)+T_{f} \\
T_{f}= \begin{cases}-\left[\left|1.175 \times 10^{6} u y+0.01(\dot{y})^{2}\right|+15000\right] \\
\times \operatorname{sgn}(\dot{y})-282.6 \dot{y}, & \dot{y} \neq 0 \\
-T_{e}, & \dot{y}=0,\left|T_{e}\right| \leq T_{s} \\
T_{s} \operatorname{sgn}\left(T_{e}\right), & \dot{y}=0,\left|T_{e}\right|>T_{s}\end{cases} \\
T_{e}=2.35 \times 10^{8}[-0.02887 \arctan (0.5886 y)+u]
\end{gathered}
$$

where

$T_{f}: \quad$ nonlinear friction force $(\mathrm{N} \mu \mathrm{m})$

$T_{e}: \quad$ external force $(\mathrm{N} \mu \mathrm{m})$

$T_{s}: \quad$ breakaway torque $(\mathrm{N} \mu \mathrm{m})$

$u$ : input signal (V)

$u_{0}: \quad$ input signal when $\dot{y}=0$

$y: \quad$ output displacement $(\mu \mathrm{m})$

$y_{0}: \quad$ output displacement when $\dot{y}=0$

The control input signal generated by the actuator is bounded at $\pm 3 \mathrm{~V}$. We denote the tracking error by $e=y_{d}-y$ where $y_{d}$ is the desired track to follow and $y$ is the actual position of the $\mathrm{R} / \mathrm{W}$ head.

\section{PROPOSED CONTROL SOLUTION: NMPC SCHEME}

The Nonlinear Model Predictive Control (NMPC) is an advanced control technique. Recently, this control scheme has gained a big interest is given to this approach due to the benefits that it offers in controlling a servo system, which are mainly robustness and ability to meet different constraints on the plant's model. The basic principle of such a control scheme is illustrated through the block diagram of Fig.2. Based on the detailed step by step description of the NMPC

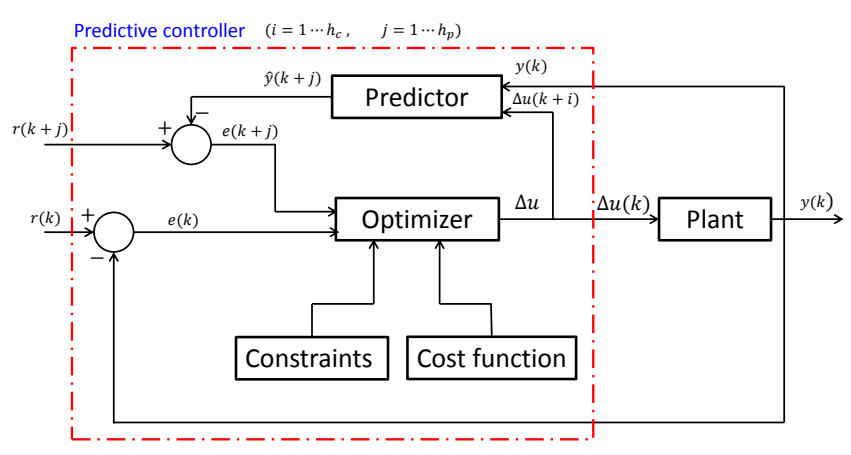

Fig. 2: Block Diagram of the NMPC controller principle

approach [19], the technique can be briefly summarized by the sequel. Consider a nonlinear discrete-time state space representation of the system to be controlled:

$$
\begin{aligned}
x(k+1) & =f(x(k), u(k)) \\
y(k) & =g(x(k))
\end{aligned}
$$

where $x(k) \in \mathbb{R}^{n_{x} \times 1}, u(k) \in \mathbb{R}^{n_{u} \times 1}$ and $y(k) \in \mathbb{R}^{n_{y} \times 1}$ denotes respectively vectors of the state, control input and output at the sampling time $k . f$ and $g$ are nonlinear functions. The control algorithm consists in the following four steps.

- step 1: At each time instant $k$, the future outputs of the plant are predicted over a predefined prediction horizon $h_{p}$. The predictions $\hat{y}(k+j \mid k)$, for $j=1, \ldots, h_{p}$ are determined using a model of the system described by equations (6)-(7) and depending on its past behaviour (previous control inputs and outputs values before the instant $k$ ) and the future control sequence $u(k+i \mid k)$, for $i=1, \ldots, h_{c}$, where $h_{c}$ is the control horizon verifying $h_{c} \leq h_{p}$.

- step 2: The optimal control sequence is determined by minimizing a performances index. This latter is often a quadratic function including the future control inputs and tracking errors defined by

$$
e(k+j \mid k)=y_{d}(k+j \mid k)-\hat{y}(k+j \mid k)
$$

where $y_{d}(k+j \mid k)$ is the reference sequence to follow assumed to be known a priori. The cost function $J$ is 
defined as follows:

$$
J=\sum_{j=1}^{h_{p}}\|e(k+j \mid k)\|_{Q}^{2}+\sum_{i=1}^{h_{c}}\|u(k+i \mid k)\|_{R}^{2}
$$

where $\|\mathbf{x}\|_{M}^{2}=\mathbf{x}^{T} M \mathbf{x}$. $Q$ and $R$ are the symmetric definite positive weighting matrices, $Q \geq 0$ and $R \geq 0$. The minimization of $J$ is often performed subject to some constraints on the control inputs and the state variables. These constraints can be presented in the form:

$$
\begin{aligned}
& \mathbb{U}:=\quad\left\{u \in \mathbb{R}^{n_{u} \times 1} \mid u_{\text {min }} \leq u \leq u_{\text {max }}\right\} \\
& \mathbb{X}:=\quad\left\{x \in \mathbb{R}^{n_{x} \times 1} \mid x_{\text {min }} \leq x \leq x_{\text {max }}\right\}
\end{aligned}
$$

$u_{\min }, u_{\max }, x_{\min }$ and $x_{\max }$ are known constant vectors. $\mathbb{U}$ and $\mathbb{X}$ are called the compact constraint subsets. The main objective of the NMPC control is to obtain the best future control sequence $[u(k \mid k), u(k+1 \mid k), \ldots, u(k+$ $\left.\left.h_{c}-1 \mid k\right)\right]$ such that the output response is the most close possible to the reference trajectory. The optimization is in general a non convex problem because of the nonlinearity of the plant model. Consequently, an online nonlinear programming is crucial to find the optimal solution.

- step 3: Only the first sample of the computed optimal control sequence is applied to the system over next sample period $[k, k+1]$.

- step 4: All the previous steps are repeated at each sampling instant.

\section{SIMULATION RESULTS: PID VERSUS NMPC}

In this section, the obtained simulation results are presented and discussed. For comparison purposes, a PID controller has also been implemented.

\section{A. Simulation environment}

Numerical simulations are conducted in Matlab 7.9 platform. For a comparative study, the PID parameters are manually tuned to obtain the best possible controller's performances. However, since the predictive algorithm is based on a nonlinear optimization problem, this makes it extremely difficult to find an analytical solution. Thus, the optimization problem for the NMPC has been solved using the MATLAB routine fmincon including the considered control constraint. The following simulation scenarios are performed:

\section{scenario 1: Nominal case with noise}

The purpose of this simulation is to show the ability of each proposed control scheme to track a variable reference trajectory ranging from $0 \mu \mathrm{m}$ to $1 \mu \mathrm{m}$. A measurement noise, which is typical a white noise due to the positionmeasurement techniques and/or sensors, is introduced to create a more realistic model. The variance of this noise is $\sigma^{2}=0.9 \times 10^{-4}(\mu \mathrm{m})^{2}$. The tuning parameters of each proposed controller are determined for this nominal case and kept unchanged for all the rest of scenarios. Table I shows a summary of the controllers' parameters. Moreover, the control input is chosen to not exceed $\pm 3 \mathrm{~V}$ which define a physical constraint on the VCM actuator. This constraint is also considered for all cases of simulation.

scenario 2: External punctual disturbance rejection

In this section, and for reasons of clarity to show the disturbance effect and the controller rejection performance, a constant reference trajectory is considered and is equal to $1 \mu \mathrm{m}$. Since a HDD always faces unexpected disruptions, an impulse disturbance with an amplitude of $0.3 \mu \mathrm{m}$ is introduced to see whether the controller is able to reject such disturbance.

scenario 3: Robustness towards parameters uncertainty (change in inertial mass)

While reading or writing data from the disk, the inertial mass of the VCM actuator of the HDD cannot be perfectly identified. Consequently, uncertainties on the latter parameter (increases of $20 \%$ and $40 \%$ with respect to the nominal value ) are introduced yielding modification on the HDD model (1). Such simulation aims to evaluate the ability of the proposed controllers to compensate these uncertainties and keep good closed-loop performance.

\begin{tabular}{|c|c|c|c|c|c|c|}
\hline \multicolumn{3}{|c|}{ PID } & \multicolumn{4}{c|}{ NMPC } \\
\hline \hline$K_{p}$ & $K_{i}$ & $K_{d}$ & $h_{p}$ & $h_{c}$ & $\mathrm{Q}$ & $\mathrm{R}$ \\
\hline 0.1 & 0.005 & 0.5 & 4 & 3 & $0.01 \times I$ & $0.01 \times I$ \\
\hline
\end{tabular}

TABLE I: Summary of the parameters of the proposed controllers

In the sequel, a comparison study of the obtained simulation results is presented, and this for the different scenarios.

\section{B. Scenario 1: Nominal case with noise}

Fig.3 and Fig.4 refer to the results obtained in this case. It is clear that NMPC controller shows better settling time performances concerning the settling time and overshoot. This controller has a very small overshoot compared with the PID controller which generates a significant one. Table II is a summary of the comparison performances.

\begin{tabular}{|c|c|c|}
\hline & Settling time (ms) & Maximun overshoot \\
\hline PID controller & 2.07 & $31 \%$ \\
\hline NMPC controller & 0.33 & $4 \%$ \\
\hline
\end{tabular}

TABLE II: Performances of the track following controllers: Nominal case with noise

\section{Scenario 2: External punctual disturbance rejection}

The objective now is to evaluate the robustness of the proposed control approaches to compensate for unexpected external punctual disturbance. As in the previous scenario, the NMPC controller shows better simulation results in terms of speed and recovery time, which can be defined as the necessary time to regain the desired trajectory after a disturbance. The obtained results are as shown in figures 5 and 6. It is clearly perceptible that the PID controller required more time to converge on the desired output and generated a significant overshoot which is not the case with the NMPC controller. With the later, the rejection of the disturbance 
is faster. This can be explained by the ability of NMPC approach to predict the future behavior of the system. In fact, when the punctual disturbance is introduced, the controller anticipates the path to follow $h_{p}$ sample time ahead, hence the convergence is faster and the recovery time is shorter than that with a PID controller. Numerical comparison is given in Table.III

\begin{tabular}{|c|c|c|}
\hline & Settling time (ms) & Recovery time (ms) \\
\hline PID controller & 1.1 & 4.1 \\
\hline NMPC controller & 0.18 & 0.4 \\
\hline
\end{tabular}

TABLE III: Performances of the track following controllers: Punctual disturbance rejection

\section{Scenario 3:Robustness against parameters uncertainty: change in the inertial mass}

Simulation results of this scenario are as shown in Fig.7 and Fig.8. For uncertainties of $20 \%$ and $40 \%$, the effect on the output response and the control input, is much more noticeable for the case of a PID controller. It is clear that the response generated significant oscillations and required longer time to converge on the disk track to be followed. However, with an NMPC controller, the effect on the system behavior with up to $40 \%$ of uncertainties is negligible. In order to test the robustness of the NMPC scheme for large uncertainties, we also introduced $100 \%$ and $200 \%$ of uncertainty on the inertial mass (see Fig.9). Here, the response is similar to that obtained with the PID controller in case of $20 \%$ and $40 \%$ of uncertainties. This shows clearly that NMPC controller is significantly more robust to compensate uncertainties and generate good performance compared to the PID controller. Table. IV summarizes some characteristics of the comparison study of this scenario.

\begin{tabular}{|c|c|c|}
\hline & Settling time (ms) & Maximum overshoot \\
\hline PID controller & 1.71 & $38 \%$ \\
\hline NMPC controller & 0.23 & $6 \%$ \\
\hline
\end{tabular}

TABLE IV: Performances of the track following controllers: Robustness towards parameter uncertainties: change in the inertial mass of $40 \%$ w.r.t nominal value

\section{CONCLUSIONS AND FUTURE WORKS}

In this paper, a NMPC approach has been investigated to control a single stage VCM actuator. This controller was observed to solve a track following problem in various operating conditions. The proposed controller was implemented as well as a PID controller (for comparison reasons) on a simulator of the system. Numerical simulations show clearly the superiority of the NMPC controller in terms of robustness and external disturbances rejection. Thus, in different operating conditions, and with the consideration of control input restrictions, the R/W head of the HDD can be maintained accurately closer to the desired track position satisfying the constraints. Further research effort will be concentrated on implementation of the proposed controller in a real HDD servo system.

\section{REFERENCES}

[1] H. Fujimoto and Y. Hori, Vibration suppression and optimal repetitive disturbance rejection control in semi-nyquist frequency region using multirate sampling control, $39^{\text {th }}$ IEEE Conference on Decision and Control, pp. 3745-3750, 2000.

[2] Y. Q. Chen, K. L. Moore, J. Yu and T. Zhang, Iterative learning control and repetitive control in hard disk drive industryA tutorial, int. journal of adaptive control and signal processing, Int. J. Adapt. Control Signal Process, 2007.

[3] M. Steinbuch, Repetitive control for systems with uncertain periodtime, Automatica, vol. 38, pp. 21032109, 2002.

[4] J. Nie, E. Sheh, and R. Horowitz, Optimal $H_{\infty}$ control for hard disk drives with an irregular sampling rate, American Control Conference, San Francisco, CA, USA, 2011.

[5] M. Hirata, M. Takiguchi, and K. Nonami, Track-following control of hard disk drives using multi-rate sampled-data $H_{\infty}$ control, Proc. 42nd Conf. on decision and control, Maui, Hawaii, USA, 2003.

[6] G. Cheng, K. Peng, M. Chen Ben,and H. Tong Lee, A microdrive track following controller design using robust and perfect tracking control with nonlinear compensation, Mechatronics 15, pp. 933948, 2005.

[7] V. Venkataramanan, Ben M. Chen, Tong H. Lee, and G. Guo, A new approach to the design of mode switching control in hard disk drive servo systems, Control Eng. Practice, vol. 10, pp. 925939, 2002.

[8] Y. Huang, W.C. Messner, and J. Steele, Feed-forward algorithms for time-optimal settling of hard disk drive servo systems, Proc 23rd Int. Conf. Ind. Electron. Contr. Instrum., New Orleans, LA, pp. 527, 1997.

[9] H. Fujimoto, Y. Hori, T. Yarnaguchi, and S. Nakagawa, Proposal of seeking control of hard disk drives based on perfect tracking control using multirate feedforward control, Proc. 6th Int. Workshop Adv. Motion Control, Nagoya, pp. 749, Japan, 2000

[10] K. Peng, B. M. Chen, G. Cheng and T. H. Lee, Modeling and compensation of nonlinearities and friction in a micro hard disk drive servo system with nonlinear feedback control, IEEE trans. on control systems technology, vol. 13, no. 5, 2005.

[11] M. Kobayashi, S. Nakagawa and H. Numasato, Adaptive control of dual-stage actuator for hard disk drives, Proc. American Control Conference, Boston, Massachusetts, 2004.

[12] C. W. Lee and S. M. Suh, Model prediction based dual-stage actuator control in discrete-time domain, IEEE Trans. on magnetics, vol. 47, no. 7, 2011.

[13] T. Sato and S. Masuda, Track-seeking control of a hard disk drive using multirate generalized predictive control to improve intersample performance, Int. journal of Innovative Computing, Information and Control, vol. 5, no. 12 (A), 2009.

[14] L. Wang,Model predictive control system design and implementation using Matlab, Advances in Industrial Control, springer, 2009.

[15] B. M. chen, T. H. Lee, K. Peng, and V. Venkataramanan, Hard Disc Drive servo sytems, 2nd edition, Advances in Industrial Control, springer, 2006.

[16] E.F. Camacho, and C.Bordons, Model predictive Control, Ed. springerverlag, London, 2004.

[17] R. Bhattacharya, G.J. Balas, M.A. Kaya,and A. Packard, Nonlinear receding horizon control of an F-16 aircraft, Journal Guidance Control and Dynamics, vol. 25(5), pp. 924-931, 2002.

[18] J.W. Eaton, and J.B. Rawlings, Model predictive control of chemical processes, Chemical Engineering Science, vol. 47(4), pp. 705-720, 1992.

[19] E.F. Camacho, and C.Bordons, Model predictive Control, Ed. springerverlag, London, 2004. 
(a)

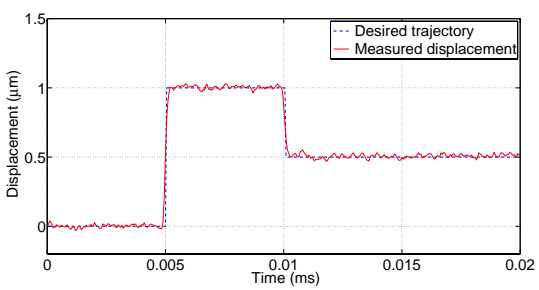

(c)

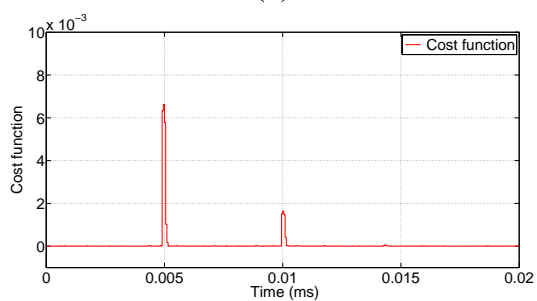

(b)

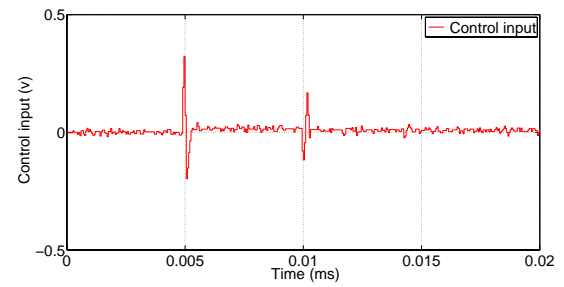

(d)

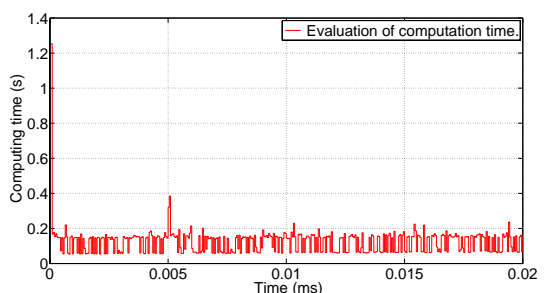

Fig. 3: Control in nominal case with noise (plots with NMPC controller): (a) output displacement,(b) control input, (c) cost function, and (d) Computing time

(a)

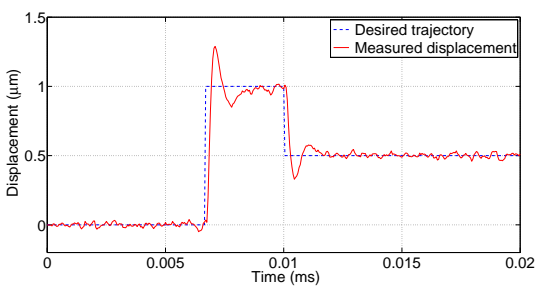

(b)

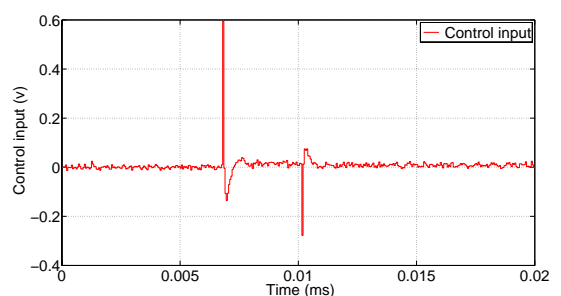

Fig. 4: Control in nominal case with noise (plots with PID controller): (a) output displacement, (b) control input

(a)

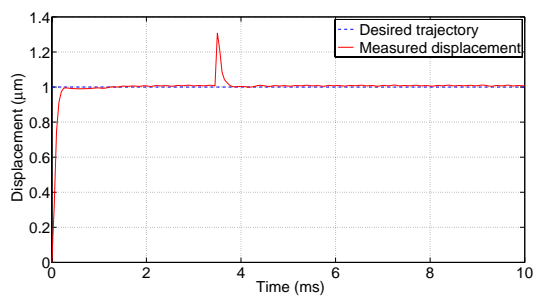

(b)

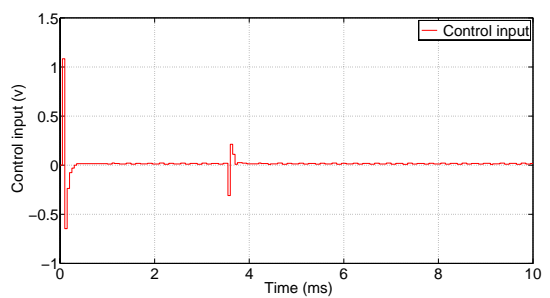

(c)

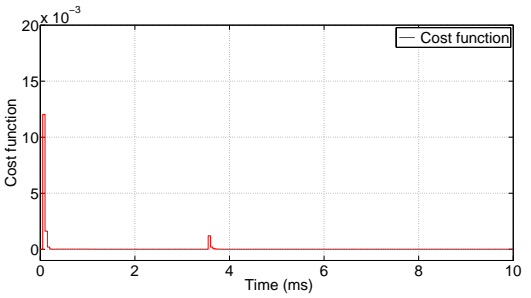

Fig. 5: Punctual disturbance rejection (plots with NMPC controller): (a) output displacement,(b) control input, and (c) cost function

(a)

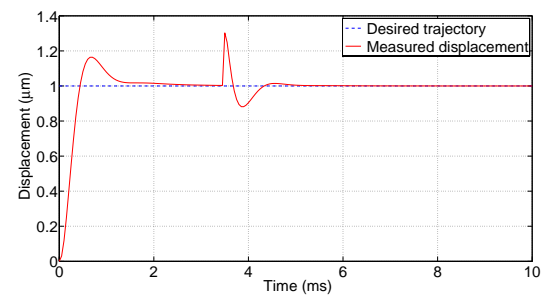

(b)

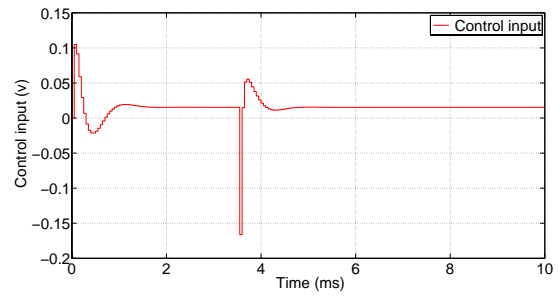

Fig. 6: Punctual disturbance rejection (plots with PID controller): (a) output displacement,(b) control input 
(a)

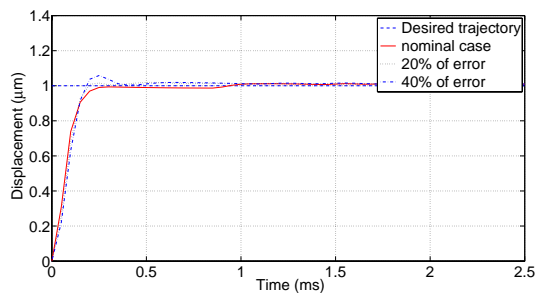

(b)

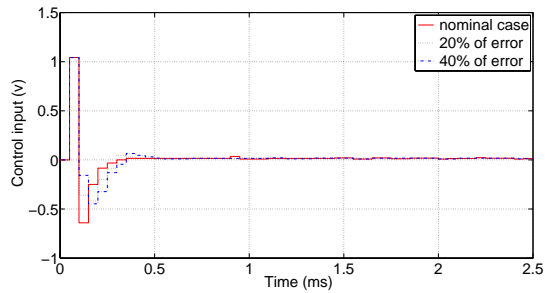

(c)

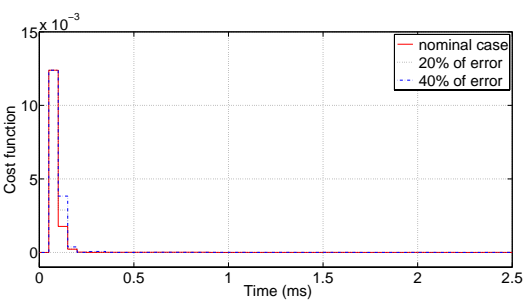

Fig. 7: Robustness towards parameters uncertainties (change of $20 \%$ and $40 \%$ in inertial mass): plots with NMPC (a) output displacement,(b) control input, and (c) cost function

(a)

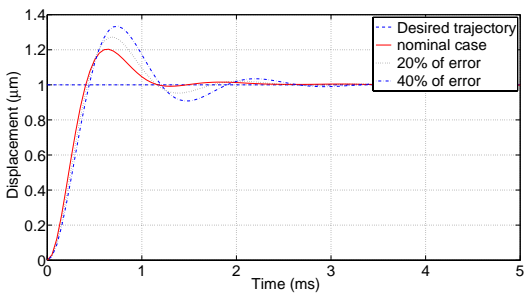

(b)

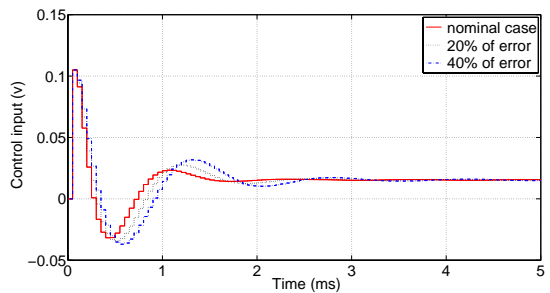

Fig. 8: Robustness towards parameters uncertainties (change of $20 \%$ and $40 \%$ in inertial mass): plots with PID (a) output displacement and (b) control input

(a)

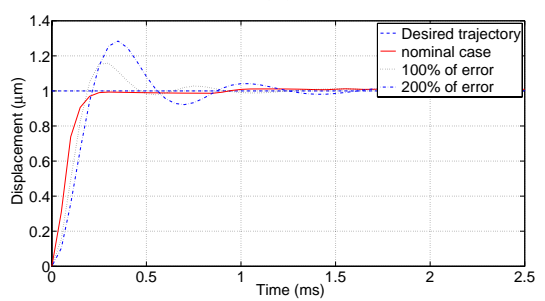

(b)

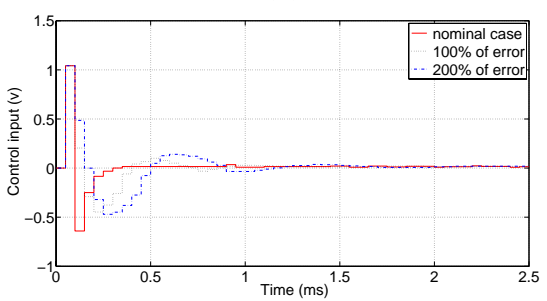

(c)

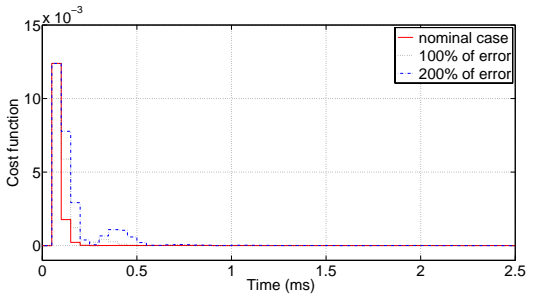

Fig. 9: Robustness towards parameters uncertainties (change of 100\% and 200\% in inertial mass): plots with NMPC (a) output displacement,(b) control input, and (c) cost function 